Portland State University

PDXScholar

6-16-2021

\title{
The Effect of Diazepam on Early Neural Stem Cells Proliferative Activity and Hippocampal-Dependent Memory after Traumatic Brain Injury
}

Van Khanh Doan

Portland State University

Follow this and additional works at: https://pdxscholar.library.pdx.edu/honorstheses

Part of the Cognitive Neuroscience Commons, Medical Biochemistry Commons, and the Other Chemicals and Drugs Commons

Let us know how access to this document benefits you.

\section{Recommended Citation}

Doan, Van Khanh, "The Effect of Diazepam on Early Neural Stem Cells Proliferative Activity and Hippocampal-Dependent Memory after Traumatic Brain Injury" (2021). University Honors Theses. Paper 1097.

https://doi.org/10.15760/honors.1124

This Thesis is brought to you for free and open access. It has been accepted for inclusion in University Honors Theses by an authorized administrator of PDXScholar. Please contact us if we can make this document more accessible: pdxscholar@pdx.edu. 
The effect of diazepam on early neural stem cells proliferative activity and hippocampal dependent memory after traumatic brain injury

\section{by \\ Van Khanh Doan}

An undergraduate honors thesis submitted in partial fulfillment of the requirements for the degree of

Bachelor of Science

in

University Honors

and

Biochemistry

Thesis Adviser

Dr. Laura Villasana

Portland State University 


\section{Abstract}

Traumatic brain injury (TBI) induces an upregulation of neurogenesis in the brain specifically in the hippocampus, an area pertaining to learning and memory formation. Although this upregulated response is intuitively thought to be restorative, previous studies show that the nascent neurons generated after TBI exhibit abnormalities, such as aberrant morphologies and enhanced migrations, which could suggest to be maladaptive. The GABA-A agonist diazepam has been shown to inhibit this upregulation in neurogenesis and normalizes dendrites after TBI. To determine whether modulation of neurogenesis with diazepam benefits or hinders cognitive recovery, C57BI/6J wild-type mice received a sham or controlled cortical impact $(\mathrm{CCl})$ injury and were administered diazepam for one week. Mice underwent the Morris Water Maze (MWM) and Reversal Morris Water Maze testing one month later to examine hippocampal-dependent memory. Diazepam was found to attenuate neurogenesis-dependent memory deficits after $\mathrm{CCl}$ in the reversal MWM test. We also examined whether modulation of post-TBI neurogenesis by diazepam is associated with attenuation of hippocampal neural stem cells (NSCs). The percent of proliferating hippocampal NSCs was not significantly different in any experimental group. Therefore, proliferative activity of hippocampal neural stem cells by diazepam was inconclusive. Further studies are needed to examine proliferating NSCs by diazepam. 


\section{Introduction}

In 2014, approximately 2.87 million people in the United States alone had traumatic brain injury (TBI)-related emergency department visits, hospitalization, and deaths (Centers for Disease Control and Prevention [CDC], 2014). Fortunately, there were only 56,800 TBI-related deaths (CDC, 2014). However, this means that the majority of the survivors have to possibly live with the lasting consequences of TBI, such as permanent cognitive impairments (Richardson et. al., 2007). A better understanding of the impact of TBI is needed to alleviate neurological consequences and improve cognitive recovery through new clinical treatments.

Neurogenesis, the regeneration of neurons, occurs throughout the lifespan of humans and is necessary for the formation of specific memories (Clelland et. al., 2009). Importantly, TBI increases neurogenesis primarily in the dentate gyrus of the hippocampus (Dash et. al., 2001; Chirumamilla et. al., 2002; Villasana et. al., 2014). The upregulation of neurogenesis after TBI could be a beneficial mechanism in which the brain attempts to repair neuronal damage sustained during TBI (Richardson et. al., 2007). However, newly immature neurons after TBI exhibit abnormal properties, such as aberrant dendritic morphology and enhanced migration within the hippocampus (Ibrahim et. al., 2016; Villasana et. al., 2015). Additionally, post-TBI neurogenesis results in long-term deficits in neural progenitor cell (NPC) proliferation and immature granule cells (IGC) (Neuberger et. al., 2017). The reduction in neurogenesis and NPC proliferative capacity in the long term, along with the aberrant characteristics of the nascent neurons, foster the importance of determining whether attenuating increases in 
post-traumatic neurogenesis potentially improves or worsens recovery of hippocampal-dependent memory after TBI.

In an attempt to answer the question of whether post-TBI neurogenesis is beneficial or detrimental, post-TBI neurogenesis is commonly manipulated to measure its cellular effect in the hippocampus and its cognitive function and recovery (Ngwenya \& Danzer, 2019). The gamma-aminobutyric acid type A (GABA-A) agonist diazepam, which is a benzodiazepine, reduces post-TBI neurogenesis and normalizes the aberrant dendritic morphology of new neurons in mice when administered for one week immediately after a controlled cortical impact $(\mathrm{CCl})$ injury in mice (Villasana et. al., 2019). In addition, the NMDA-R antagonist ketamine also reduces post-TBI neurogenesis and in conjunction improves strategy flexibility in the hippocampal-dependent water maze test (Peters et. al., 2018). Therefore, the ability of diazepam to inhibit post-TBI neurogenesis and rectify the aberrant dendritic morphology makes it a potentially relevant tool in post-TBI cognitive recovery, as seen with ketamine. However, neither the association between diazepam-induced neurogenesis reduction and cognitive recovery after TBI nor its mechanism of reduction have yet to be examined.

The change in neurogenesis after TBI could be due to many factors including neural stem cell (NSC) proliferation, cell survival, and differentiation, as well as changes in the neural environment. Massive change in the extracellular flow of ions and neurotransmitters following a TBI could affect cell survival by inducing an excitotoxicity environment, which can cause metabolic stress and cause excitotoxic neuronal death (Katayama et. al., 1990). NSCs reside in the dentate gyrus of the hippocampus and can 
proliferate and differentiate into two cell types: astrocytes or neurons (Bond et. al., 2015). It is possible that the differentiation of NSC could change in the presence of diazepam where neuronal differentiation is suppressed or the level of differentiation of other cell types is increased. Diazepam has also been shown to block hippocampal activity and NSC proliferation after a middle cerebral artery occlusion (MCAO), a stroke injury model, which also increases neurogenesis in mice (Nochi et. al., 2013). Therefore, similar to $\mathrm{MCAO}$, in the $\mathrm{CCl}$ injury model, diazepam treatment may inhibit the transient post-TBI neurogenesis via the inhibition of NSC proliferation.

Benzodiazepines are often administered to severe-TBI patients upon hospitalization for injury management (Flower \& Hellings, 2012). The effects of benzodiazepines act transiently to reduce the consciousness of the patients, elevated intracranial pressure, and the seizure susceptibility (Flower \& Hellings, 2012). As a possible agent employed to provide early medical care, it is additionally important to determine the mechanism of diazepam on post-TBI neurogenesis and whether the administration of diazepam can play a role in long-term cognitive recovery. In this study, the effects of diazepam administration after $\mathrm{CCl}$ on hippocampal-dependent memory were examined. We also examined whether diazepam administration immediately after $\mathrm{CCl}$ attenuated the proliferation of NSCs.

\section{Method}

\section{Animals}

All mouse handling and procedures were performed in accordance with National Institutes of Health guidelines and were in compliance with Oregon Health and Science and Legacy Research Institute Institutional Animal Care and Use Committee approved 
protocols. Seven-week old male and female C57BI/6J wild-type mice were purchased from Jackson Laboratories and allowed to acclimate one week prior to surgeries. A total of 28 surviving mice were used for the behavioral assays. For the analysis of NSC proliferation, brain tissue from 16 mice from the previous study (Villasana et al., 2019) were used.

\section{Controlled cortical impact injury and osmotic pump implantation}

Controlled cortical impact $(\mathrm{CCl})$ was used as the mouse TBI model. There were four groups of mice: sham/vehicle (4 males, 4 females), $\mathrm{CCl} /$ vehicle (3 males, 3 females), sham/diazepam (4 males, 3 females), and CCI/diazepam (4 males, 3 females). Littermates were used for each group. Four mice had to be euthanized due to suture complications (28 mice remaining). Approximately an hour before surgery, all mice received $0.2 \mathrm{~mL}$ of Children's Tylenol (orally). During the injury surgery, all mice were anesthetized with $2 \%$ isoflurane (continuously inhaled) and placed on a heated stereotaxic apparatus. Their heads and left shoulder blades were shaved and sterilized with $10 \%$ betadine followed by $2 \%$ lidocaine. An incision was made along the midline of the scalp followed by a $4 \mathrm{~mm}$ lateral (left) craniotomy between bregma and lambda leaving the dura mater intact. At the exposed area, a $3 \mathrm{~mm}$ stainless steel piston attached to an electromagnetic impactor (Leica Microsystems, Germany) was used to make a $0.9 \mathrm{~mm}$ deformation at a $4.67 \mathrm{~m} / \mathrm{s}$ velocity with a dwell time of $300 \mathrm{~ms}$. Sham mice received the same procedure minus the craniotomy and injury. The incision was sutured and osmotic pumps (Alzet; Cupertino, CA) containing either vehicle (1:1 DMSO/propylene glycol) or diazepam (15 mg/kg/day) were subcutaneously implanted over the left shoulder blade immediately after the sham or $\mathrm{CCl}$ injury. Mice were ear 
punched for identification and allowed to recover in a warm padded chamber for 45 minutes prior to conducting a brief neurologic severity assessment. All mice were given Tylenol twice daily for 2 days after surgery. Osmotic pumps were removed one week later via the removal and reinstatement of sutures at the incision and saved to confirm the delivery rate.

\section{BrdU protocol}

Behavioral mice received 3 intraperitoneal injections (2 hours apart) of BrdU (50 mg/kg dissolved in saline), beginning 2 days after $\mathrm{CCl}$ or sham. BrdU provides the labeling of proliferating cells (Cameron \& McKay, 2001). Their histological examination of NSCs using BrdU is not included in this study.

\section{Assessment of neural stem cell proliferation}

Tissue from sham or $\mathrm{CCl}$ treated mice with or without diazepam was obtained from a previous study conducted at Oregon Health and Science University (Villasana et al., 2019). In that study, proliferation in general was assessed with only BrdU or Ki67 in response to diazepam 3 days after the injury. In this study, with the same samples, the proliferation of NSCs was assessed using Ki67 coupled with GFAP and Sox2. The number of mice brain samples used in the following experimental group were 4 sham/veh, $4 \mathrm{CCl} / \mathrm{veh}, 4$ sham/dzp, $4 \mathrm{CCl} / \mathrm{dzp}$ from the previous study (Villasana et. al., 2019). 


\section{Immunohistochemistry}

Immunohistochemistry $(\mathrm{IHC})$ was performed as previously described (Villasana et. al., 2019) with some modifications. To perform IHC with Ki67/Sox2/GFAP/DAPI, four brain sections containing the hippocampus (two dorsal, two ventral) were obtained from each mouse. The brain sections were washed with $0.5 \mathrm{~mL}$ PBST for 10 minutes five times. The sections were blocked with 5\% normal donkey serum (NDS) in PBST for 60 minutes then incubated at $4{ }^{\circ} \mathrm{C}$ overnight with primary antibodies diluted with $1.5 \%$ NDS. The primary antibodies used were rabbit anti-Ki67 (1:200), rat anti-Sox2 (1:400), and mouse anti-GFAP (1:400). The sections were washed with PBST for 10 minutes three times and incubated with the secondary antibodies for 2-3 hours. The secondary antibodies used were donkey anti-rabbit (1:500, Alexa fluor 488), donkey anti-rat (1:500, Alexa fluor 555), and anti-mouse (1:500, Alexa fluor 647). The sections were then washed with PBST for 10 minutes three times and incubated in DAPI in PBST $(1: 10,000)$ for 20 minutes. Finally, the sections were washed with PBST for 10 minutes two times and mounted on slides with Fluoromount G (Southern Biotech).

\section{Confocal microscopy and imaging analyses}

The dentate gyrus of the hippocampus was imaged with a Leica TCS SPE-II confocal microscope using a $20 \times 0.8$ lens. All subsequent analyses were performed on coded images with ImageJ software. The targeted cells were only counted in the granular cell layer (GCL) and the subgranular zone (SGZ) of the dentate gyrus. Specifically, Ki67 labels cells undergoing G1, S, G2, and M phase (Tanaka et. al., 2011). Sox2 labels neural stem cells (NSCs) and astrocytes. GFAP labels the processes of the astrocytes and NSCs. In Ki67/Sox2/GFAP stained sections, NSCs were identified by the 
colabeling of Sox2 and GFAP with a singular process from the cell body (versus astrocytes with multiple processes). Of these NSCs, proliferation was identified by the additional labeling with Ki67. The volume of the GCL was measured by collecting the depth (z-stack) of the image (ranges from 13-26 $\mu \mathrm{m}$ ) and the area of the GCL. The cell density was measured by taking the number of cells over the volume of GCL $\left(\#\right.$ cells $\left./ \mathrm{mm}^{3}\right)$. The percentage of proliferating NSCs were calculated by taking the number of proliferating NSCs (KI67/Sox2/GFAP) over the total number of NSCs (Sox2/GFAP).

\section{Behavioral analyses}

Behavioral testing began 4 weeks after surgeries. One hour prior to conducting the water maze test, mice were temporarily singly housed in cages, which sat halfway on top of heating pads. A $122 \mathrm{~cm}$ circular pool was filled with water $\left(20^{\circ} \mathrm{C} \pm 1\right)$ and made opaque using white chalk. The water maze test was conducted with 2 sessions of training each day with each session consisting of 3 trials. The first three days out of 7 days, mice were trained to locate the platform within the pool. Distal cues were placed around the room, and consisted of evenly distanced black rectangle boards with either a circle, a horizontal line, a vertical line, or no line through the middle. Mice were placed in a pseudorandom location within the pool and were given 60 seconds to swim and locate the "hidden" platform which sat $3 \mathrm{~cm}$ below the water. If they did not find the platform within 60 seconds, they were hand guided to the platform location and allowed to sit on it for 3 seconds before removing them. If they did find the platform within 60 seconds, they were allowed to remain on it for 3 seconds as well before removing them. On the 4th day (7th session), a probe trial was conducted to assess the spatial memory 
retention of the mice. In this trial, the platform was removed, and the bias for the location of the platform was assessed. To mitigate any potential extinction effects of the probe trial, a reinforcement session with the platform returned to its original location was conducted immediately after the probe trial.

\section{Reversal Water Maze}

On day 5 and 6 (session 8-11), mice were trained to locate a new location of the platform in a new quadrant of the pool. A probe trial was conducted 24 hours after each of these training days. Mice were euthanized 90 minutes after the last reversal probe trial.

\section{Statistical analyses}

For all analyses, the data was first assessed for normality to determine use of parametric versus non-parametric tests. For water maze analyses, a 3-way repeated measures ANOVA with injury drug and sex as between subject variables and session as the within subject factor was used to assess the learning curves. For the probe trials, a 3-way ANOVA was used to compare group differences in the cumulative distance swam from the platform. The proliferation of NSCs was compared between groups using a 3-way ANOVA. Fisher's post-hoc test was used where appropriate. Figures were created using Prism GraphPad Software, La Joylla, CA) and statistics were conducted using SPSS Software (IBM SPSS, Armonk NY).

\section{Results}

Diazepam improves spatial acquisition during the learning phase of the water maze test after $\mathrm{CCl}$ 
To determine the effect of diazepam after $\mathrm{CCl}$ on cognitive recovery, mice underwent Morris water maze behavioral testing 4 weeks after injury. The performance on the Morris water maze test depends on hippocampal function, which allows the mice to form a spatial map of the room using visual cues in order to locate the escape platform hidden just underneath the water (Morris, 1984). Changing the location of the hidden platform (reversal task) requires the mice to integrate overlapping context and change search strategies. This type of learning and memory is sensitive to changes in neurogenesis (Garthe \& Kempermann, 2013). Mice were first trained to locate the platform in one quadrant and the distance from the target platform was calculated to measure their performance (Figure 1A). As the number of sessions increased, the distance from the target platform decreased for all groups of mice, illustrating they were all able to learn the hidden platform location. On average across all training sessions, however, $\mathrm{CCl} /$ vehicle treated mice performed significantly worse $(\mathrm{p}<0.05)$. In contrast, the CCl/Diazepam(dzp) treated mice showed no differences from the sham/veh or the sham/dzp group. However, by the end of the last session, all mice were able to locate the hidden platform without group differences.

In session 7, a probe trial was conducted in which the platform was removed and the distance the mice swam from the learned location of the platform was calculated (Figure 1B). The $\mathrm{CCl} /$ veh group appeared to perform worse compared to the sham/veh group but group differences did not reach statistical differences. When mice cross the learned location of the platform, a platform crossing was recorded (Figure 1C). Higher the platform crossings are an indication of retained memory. Likewise, the $\mathrm{CCl} / v e h-t r e a t e d$ mice showed a similar trend to perform worse with the least number of 
platform crossings compared to the rest of the groups but this did not reach statistical significance. Because all the mice groups were able to perform similarly to each other with no statistical significance at the end of training and the probe trial, they were qualified to be tested in the reversal water maze.

\section{Diazepam ameliorates the CCl-induced memory impairment in the water maze reversal}

In conjunction with the first platform location during the water maze test, mice were in addition subjected to a reversal Morris water maze test. In the reversal Morris water maze test, mice had to learn a new platform location in the opposite quadrant from the first. The cues around the room remained the same, but mice had to integrate the overlapping cues for the 2 nd platform from the first. This type of integration and memory is neurogenesis-dependent (Garthe \& Kempermann, 2013).

In Figure $2(D)$, the training for the reversal water maze was conducted from session 8 to session 11. As the number of sessions increased, the distance from platform decreased, showing that the mice were able to learn the new location of the second platform. However, the $\mathrm{CCl} /$ veh-treated mice performed significantly worse than all other groups in sessions 10 and $11(p<0.05$, session $x$ drug $x$ injury interaction). In contrast, the $\mathrm{CCl} / \mathrm{dzp}$ group had similar distances from the platform as that of sham/veh and sham/dzp mice.

In session 12, a probe trial for the reversal maze water test was conducted to examine the ability of mice to retain the spatial memory for the 2 nd platform. The memory retention for the second platform was measured by the distance swam from the 2nd platform (Figure 2E). The distance swam from the 1st platform was also measured. 
The $\mathrm{CCl} /$ veh mice were impaired compared to the other groups. (drug $\mathrm{x}$ injury interaction, $p<0.05)$. They performed significantly worse than sham $/ v e h$ mice $(p=0.01)$ and $\mathrm{CCl} / \mathrm{dzp}(\mathrm{p}=0.01)$ by swimming the farthest from the location of the platform. In the same figure, there was no statistical significance among the groups in distance swam from the 1st platform. Similar group trends were observed on the platform crossings, although these did not reach statistical significance (Figure 2F). Sham/veh, sham/dzp, and $\mathrm{CCl} / \mathrm{dzp}$ groups showed both platform crossings for the 1 st platform and the 2 nd platform, but the platform crossings were greater for the 2nd platform. Meanwhile, the $\mathrm{CCl} /$ veh group had the least platform crossings for the 2 nd platform and no platform crossings for the 1st platform. Surprisingly, the $\mathrm{CCl} / \mathrm{dzp}$ group showed the most platform crossings between the 1 st platform and the 2 nd platform, being able to locate the 2 nd platform and retain the memory of the 1st platform the most, but these did not reach a statistical difference and was considered a trend only.

\section{Diazepam does not inhibit the proliferation of neural stem cells after $\mathrm{CCl}$}

To investigate the mechanism in which diazepam reduces neurogenesis, the proliferation of neural stem cells was examined using cell markers Sox2,GFAP, and Ki67. A reduction in the proliferation of NSCs could result in the reduced production of neurons. Figure 3 (Right) shows a slight increase in NSC proliferation in $\mathrm{CCl} /$ veh group compared to sham/veh group, which is congruent to the upregulation of neurogenesis, but this did not reach significance as neither did the CCl/dzp group. In addition, the figure shows a slight decrease of proliferating NSCs between $\mathrm{CCl} / v e h$ and $\mathrm{CCl} / \mathrm{dzp}$, which could explain the inhibition of post-TBI neurogenesis by diazepam, but this did not reach statistical significance. 


\section{Discussion}

Diazepam, a GABA-A agonist, plays an inhibitory role in the central nervous system by increasing the opening of chloride channels (Study \& Barker, 1981) and thus increasing the likelihood of hyperpolarization (inhibition) of neural cells (Pontes et. al., 2013). GABAergic regulation within the brain modulates the proliferation of cells as well as neuronal maturation and dendritic development (Ge et. al., 2005; Duveau et. al., 2011), and the GABA-A agonist diazepam rectifies the aberrant dendritic morphologies associated with post-TBI neurogenesis (Villasana et. al., 2019). The administration of diazepam has previously been shown to block hippocampal activity as well as NSC proliferation but in a different injury model (Nochi et. al., 2013). Therefore, to observe the inhibitory nature of diazepam in reducing neurogenesis after $\mathrm{CCl}$, the level of NSCs proliferation was histologically examined in this study as the suspected inhibitory mechanism. To gain a better understanding of how modulation of post-traumatic neurogenesis by diazepam may affect recovery, behavioral outcomes of the water maze test were also examined.

We found that the administration of diazepam after $\mathrm{CCl}$ prevented deficits in the learning acquisition during standard reference memory in the water maze test. We also found that it prevented deficits in strategy flexibility during the reversal water maze test. Although we found group trends in the proliferative behavior of the NSCs, differences were not enough to reach significance, and therefore no conclusion could be made.

\section{Rescued spatial memory acquisition with diazepam}

Despite all groups improving their ability to locate the first platform, $\mathrm{CCl} / \mathrm{veh}$ treated mice performed worse across the average of the training sessions whereas 
$\mathrm{CCl} / \mathrm{dzp}$ treated mice did not and emulated results similar to the control groups. This suggests that administering diazepam after injury is beneficial for spatial acquisition. However, the difference in neurogenesis between groups at this point was not histologically assessed in this study, and therefore how this protection is associated with post-TBI neurogenesis has yet to be determined.

In the second training for the reversal water maze test, mice treated with vehicle after $\mathrm{CCl}$ performed worse in learning the location of the second platform over repeated sessions. Mice treated with diazepam after $\mathrm{CCl}$ however performed as well as the sham/veh treated mice. The difference between the first training for the first platform and the second training for the 2 nd platform is that $\mathrm{CCl} /$ veh treated mice were able to retain memories of the first platform at the end despite difficulty at first, whereas $\mathrm{CCl} /$ veh treated mice in the reversal learning phase stayed impaired throughout. This suggests that the $\mathrm{CCl}$ injury did not impair the hippocampal-dependent ability to form a spatial map but did impair the neurogenesis-dependent memory to integrate overlapping information associated with the same spatial map. The administration of diazepam after $\mathrm{CCl}$ rectified these learning deficits in both trainings and returned learning to a normal level seen in the non-injured mice treated with vehicle.

\section{Normal spatial memory retention during the 1st probe trial with and without}

\section{diazepam}

Before the 1st probe trial, mice had learned the location of the first platform with associated cues around the pool. In the probe trial, the platform is removed, and the mice must be able to orient themselves spatially using the associated cues to find the location of the platform. Although no statistical difference was found in the distance to 
platform and platform crossings data, $\mathrm{CCl} /$ veh treated mice tended to perform the worst, and mice treated with diazepam after $\mathrm{CCl}$ tended to perform better. No statistical differences among the groups suggest that hippocampal-dependent spatial memory retention during this probe trial was not affected by $\mathrm{CCl}$ and diazepam, even if initial memory acquisition was negatively impacted during training by $\mathrm{CCl}$ and improved with diazepam.

\section{Improved neurogenesis-dependent memory during the 2nd probe trial with}

\section{diazepam}

The reversal Morris water maze is used to further test neurogenesis-sensitive memory (Clelland et. al., 2009; Garthe et. al., 2013). The test requires integration between similar cues in the new (2nd) platform versus cues in the old (1st) platform. The $\mathrm{CCl}$ injury impacted the ability to integrate the memory of the 2 nd platform after memory of the 1 st platform. The $\mathrm{CCl}$ only mice performed the worst in the reversal water maze test, but the mice treated with diazepam after $\mathrm{CCl}$ performed better and similarly to the control sham/veh mice. Interestingly, the non-injured mice treated with diazepam did not show a significant difference in the distance from platform 2 compared to the $\mathrm{CCl}$ only group. They had a higher distance swam than sham/veh and $\mathrm{CCl} / \mathrm{dzp}$ but lower than $\mathrm{CCl}$ only group. This could suggest that diazepam hindered memory integration of the second platform in non-injured mice, but this did not reach statistical significance. One experimental problem could be the rigorousness of the contextual cues. The cues employed for both water maze tests may have been so similar that the mice had trouble differentiating the cues themselves and the cues associated with each platform. This was done intentionally to make the test more challenging by requiring 
pattern separation, a neurogenesis-sensitive type of learning (Clelland et. al., 2009;

Garthe et. al., 2013)

The link between NSCs proliferative activity and modulation of post-CCI neurogenesis by diazepam requires further testing

Neural stem cells are responsible for the production of astrocytes and neurons within the subgranular zone (SGZ) of the dentate gyrus (Bond, 2015). Therefore, it was suspected that the reduction in hippocampal-neurogenesis by diazepam was due to the reduction in the proliferative activity of SGZ NSCs. In this study, no links were observed. However, this aspect of the study warrants further investigation. Because of differences in tissue preservation, we were restricted to a limited number of samples. A greater sample size is needed to definitively investigate NSCs. In addition, positive proliferating NSCs stipulated the triple-labeling of Ki67, Sox2, and GFAP. Better utilization of different antibodies to improve the identification of proliferating and non-proliferating NSCs may reduce cell counting errors by the researcher.

It is also possible that diazepam may act through on another stage of neurogenesis to inhibit post-TBI neurogenesis. Before NSCs differentiate into a neuronal cell, they give rise to intermediate progenitor cells (IPCs) which will then become neurons (Kriegstein \& Alvarez-Buylla, 2011; Harris et. al., 2016). Thus, it is possible that the effect of diazepam could be at the level of IPCs. Further IHC studies can investigate IPCs proliferative activity with diazepam to determine the mechanism by which diazepam reduces post-CCl neurogenesis.

\section{Conclusion:}


The administration of diazepam after traumatic brain injury rectifies learning deficits in the Morris water maze test and the reversal water maze test one month after injury. This is followed up with an improvement in neurogenesis-dependent memory in the reversal water maze probe trial. Additional studies focusing on the cellular mechanism of diazepam in brain injury are needed to gain a better understanding of the physiological interaction between the TBI and the diazepam. In addition, behavioral studies including other cognitive domains are needed to better understand how diazepam affects cognitive recovery. These results provide a foundation for further examining the clinical utility of benzodiazepines on cognitive recovery after brain injury.

\section{Acknowledgements}

I would like to thank Dr. Laura Villasana, my thesis advisor, for the project guidance and for being the most influential person in my undergraduate career; Ariel Weingarten, a research assistant in the Villasana lab, for her hard work in conducting the experiments in place of me during the Covid-based lab restrictions; and SreeNeha Yeturu, a fellow research intern, for her assistance in data analysis.

The material in this study is supported and conducted at Oregon Health and Science University and Legacy Research Institute. 


\section{References}

Bond, A., Ming, G., \& Song H. (2015) Adult Mammalian neural stem cells and neurogenesis: Five Decades later. Cell Stem Cell. NIH DOI: https://pubmed.ncbi.nlm.nih.gov/26431181/

Centers for Disease Control and Prevention (2014). Surveillance report of traumatic brain injury-related emergency visits, hospitalizations, and deaths. U.S. Department of Health and Human Services. DOI: https://www.cdc.gov/traumaticbraininjury/data/

Chirumamilla, S., Sun, D., Bullock, M., \& Colello, R. (2002). Traumatic brain injury induced cell proliferation in the adult mammalian central nervous system. Journal of Neurotrauma. NIH. DOI: https://pubmed.ncbi.nlm.nih.gov/12165131/

Clelland, C., Choi M., Romberg C., Clemenson, G., Fragniere, A., Tyers, P., Jessberger, S., Saksida, L., Barker, R., \& Bussey, T. (2009).A functional role for adult hippocampal neurogenesis in spatial pattern separation. National Institutes of Health. Science. DOI: https://www.ncbi.nlm.nih.gov/pmc/articles/PMC2997634/pdf/nihms-243000.pdf

Dash, P., Mach S., and Moore A. (2001). Enhanced neurogenesis in the rodent hippocampus following traumatic brain injury. Journal of Neuroscience Research. Wiley Online Library. DOI: https://onlinelibrary.wiley.com/doi/abs/10.1002/1097-4547(20010215)63:4\%3C313::AID-JNR102 5\%3E3.0.CO;2-4

Duveau, V., Laustela, S., Gianolini, F., Vogt, K., Keist, C., Homanics, G., Rudolph, U., \& Fritschy, J. (2011). Spatiotemporal specificity of GABAA receptor-mediated regulation of adult hippocampal neurogenesis. European Journal of Neuroscience. NIH. DOI: https://pubmed.ncbi.nlm.nih.gov/21722213/

Flower, O. \& Hellings S. (2012). Sedation in traumatic brain injury. Emergency Medicine International. NIH. DOI: https://pubmed.ncbi.nlm.nih.gov/23050154/

Garthe, A. \& Kempermann G. (2013). An old test for new neurons: refining the Morris water maze to study the functional relevance of adult hippocampal neurogenesis. Frontiers in Neuroscience. NIH. DOI: https://pubmed.ncbi.nlm.nih.gov/23653589/

Ge, S., Goh, E., Sailor, K., Kitabatake, Y., Ming, G., \& Song, H. (2005). GABA regulates synaptic integration of newly generated neurons in the adult brain. Nature. DOI:

https://www.nature.com/articles/nature04404

Harris, L., Zalucki, O., Gobius, I., McDonald, H., Osinki, J., Harvey, T., Essebier, A., Vidovic, D., Brune, T., Heng, J., Richards, L., Gronostajski, R., \& Piper, M. (2016). Transcriptional regulation of intermediate progenitor cell generation during hippocampal development. Development. DOI: https://journals.biologists.com/dev/article/143/24/4620/47628/Transcriptional-regulation-of-inter $\underline{\text { mediate }}$ 
Ibrahim, S., Hu, W., Wang, X., Xiang, G., He, C., \& Chen, J. (2016). Traumatic brain injury causes aberrant migration of adult-born neurons in the hippocampus. Nature. Scientific Reports. DOI: https://www.nature.com/articles/srep21793

Katayama, Y., Becker, D., Tamura, T., \& Hovda, D. (1990). Massive increases in extracellular potassium and the indiscriminate release of glutamate following concussive brain injury. Journal of Neuroscience. NIH. DOI: https://pubmed.ncbi.nlm.nih.gov/1977896/

Kriegstein, A. \& Alvarez-Buylla A. (2011). The glial nature of embryonic and adult neural stem cells. Annual Review of Neuroscience. NCBI. DOI:

https://www.ncbi.nlm.nih.gov/pmc/articles/PMC3086722/

Morris, R. (1984). Development of a water-maze procedure for studying spatial learning in the rat. Journal of Neuroscience Methods. Science Direct. DOI:

https://www.sciencedirect.com/science/article/abs/pii/0165027084900074?via\%3Dihub

Neuberger, E., Swietek, B., Corrubia, L., Prasanna, A., \& Santhakumar, V. (2017). Enhanced

Dentate neurogenesis after brain injury undermines long-term neurogenic potential and promotes seizure susceptibility. Stem Cell Reports. DOI:

https://www.sciencedirect.com/science/article/pii/S2213671117303259

Ngwenya, L., \& Danzer, S. (2019). Impact of Traumatic Brain Injury on Neurogenesis. Frontiers in Neuroscience. DOI: https://www.frontiersin.org/articles/10.3389/fnins.2018.01014/full

Nochi, R., Kaneko, J., Okada, N., Terazono, Y., Matani, A., \& Hisatsune, T. (2013). Diazepam treatment blocks the elevation of hippocampal activity and the accelerated proliferation of hippocampal neural stem cells after focal cerebral ischemia in mice. Journal of Neuroscience. Wiley Online Library. DOI: https://onlinelibrary.wiley.com/doi/epdf/10.1002/jnr.23264

Peters, A., Villasana, L., \& Schnell, E. (2018). Ketamine alters hippocampal cell proliferation and improves learning in mice after traumatic brain injury. OHSU. DOI:

https://ohsu.pure.elsevier.com/en/publications/ketamine-alters-hippocampal-cell-proliferation-an d-improves-learn

Pontes, A., Zhang, Y., \& Hu, W. (2013). Novel functions of GABA signaling in adult neurogenesis. Frontiers in Biology. DOI:

https://link.springer.com/article/10.1007/s11515-013-1270-2\#citeas

Richardson, R., Sun, D., \& Bullock M. (2007). Neurogenesis After Traumatic Injury.

Neurosurgery Clinics of North America. DOI:

https://www.researchgate.net/publication/6557822_Neurogenesis_After_Traumatic_Brain_Injury 
Study, R., \& Barker, J. (1981). Diazepam and (--)-pentobarbital: fluctuation analysis reveals different mechanisms for potentiation of gamma-aminobutyric acid responses in cultured central neurons. PNAS. NCBI. DOI: https://www.ncbi.nlm.nih.gov/pmc/articles/PMC349220/

Tanaka, R., Tainaka, M., Ota, T., Mizuguchi, N., Kato, H., Urabe, S., Chen, Y., Fustin, J.M., Yamaguchi Y., Doi, M., Hamada, S., \& Okamura, H. (2011). Accurate determination of S-phase fraction in proliferative cells by dual fluorescence and peroxidase immunohistochemistry with 5-bromo-2'-deoxyuridine (BrdU) and Ki67 antibodies. Journal of Histochemistry \& Cytochemistry. NIH. DOI: https://pubmed.ncbi.nlm.nih.gov/21551319/

Villasana, L., Westbrook, G., \& Schnell, E. (2014). Neurologic impairment following closed head injury predicts post-traumatic neurogenesis. OHSU. DOI:

https://ohsu.pure.elsevier.com/en/publications/neurologic-impairment-following-closed-head-inju ry-predicts-post--2

Villasana, L., Peters, A., McCallum, R., Liu, C., \& Schnell, E. (2019). Diazepam inhibits post-traumatic neurogenesis and blocks aberrant dendritic development. OHSU. DOI:

https://ohsu.pure.elsevier.com/en/publications/diazepam-inhibits-post-traumatic-neurogenesis-a nd-blocks-aberrant 


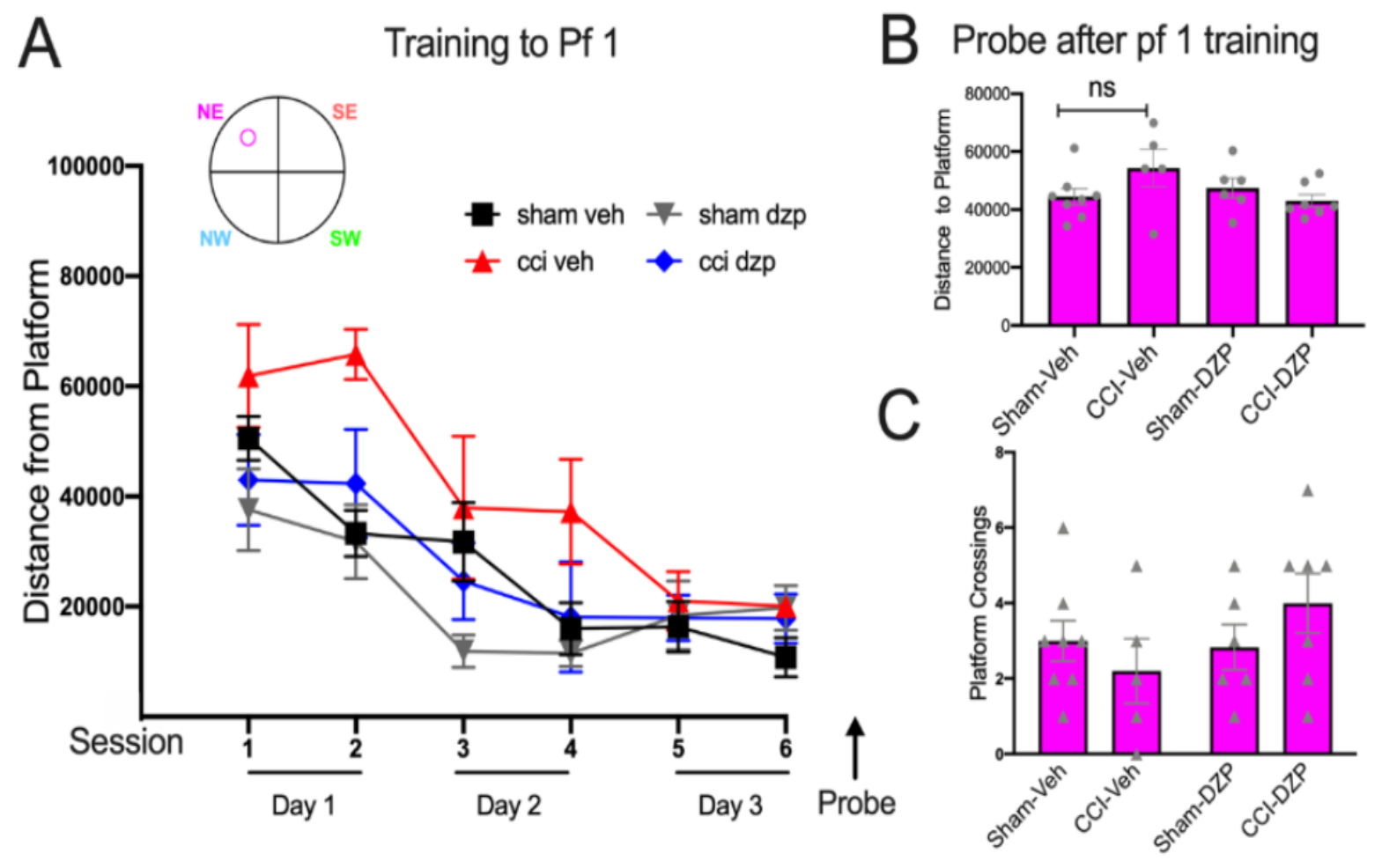

Figure 1:Diazepam improved performance of $\mathrm{CCl}$ treated mice during spatial acquisition. (A) Learning curves during spatial acquisition of the first hidden platform as measured how close mice swam to the location of the platform. $\mathrm{CCl} /$ vehicle-treated mice performed significantly worse across the average of the sessions $(p<0.05)$. $(B-C)$ Probe trial performance 24-hour after session 6. Group trends show no significance. 


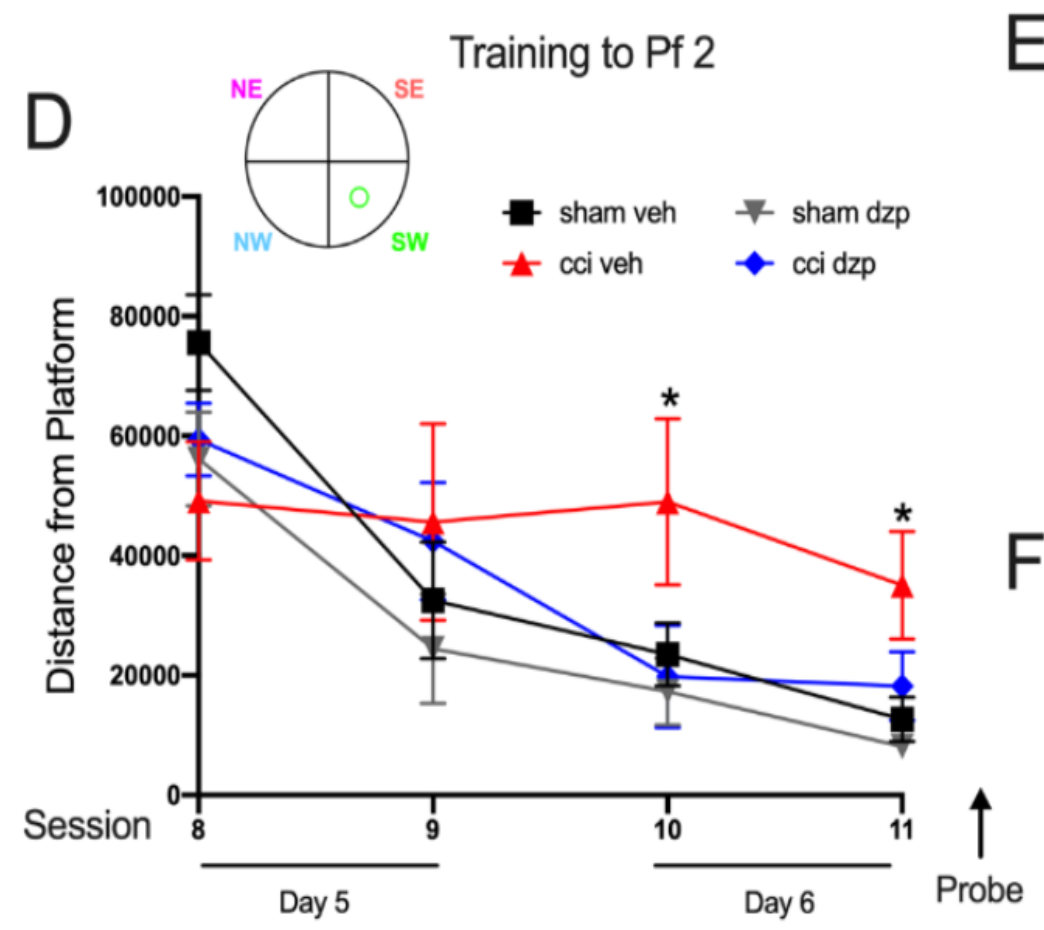

E Probe after reversal training

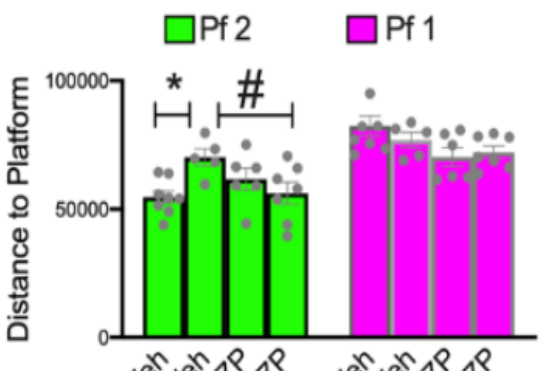

F
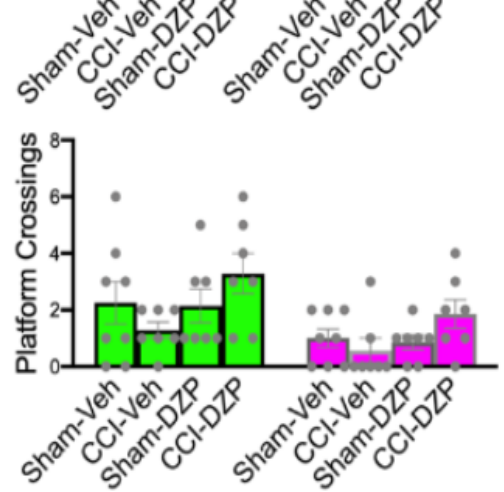

Figure 2: Diazepam rescues CCl-induced deficits in the reversal water maze test. (D) Learning curves for the 2 nd platform location. $\mathrm{CCl} /$ veh treated mice were impaired compared to controls in sessions 10 and $11(p<0.05)$ whereas $\mathrm{CCl} / \mathrm{dzp}$ treated mice were not $\left(F_{2.7,60.4}=2.98 ; p<0.05\right.$, session $x$ injury $x$ treatment $)$. $(E)$ During the probe trial for the 2nd platform, $\mathrm{CCl} /$ veh treated mice swam farther from the second location of the platform compared to sham/veh and $\mathrm{CCl} / \mathrm{dzp}$ treated mice $\left(F_{1,22}=8.23 ; p<0.01\right.$, injury $x$ drug interaction; ${ }^{*} p<0.01$ vs sham/veh; ${ }^{\#} p<0.05$ vs CCl/dzp). (F) Similar trends were observed in platform crossings. 

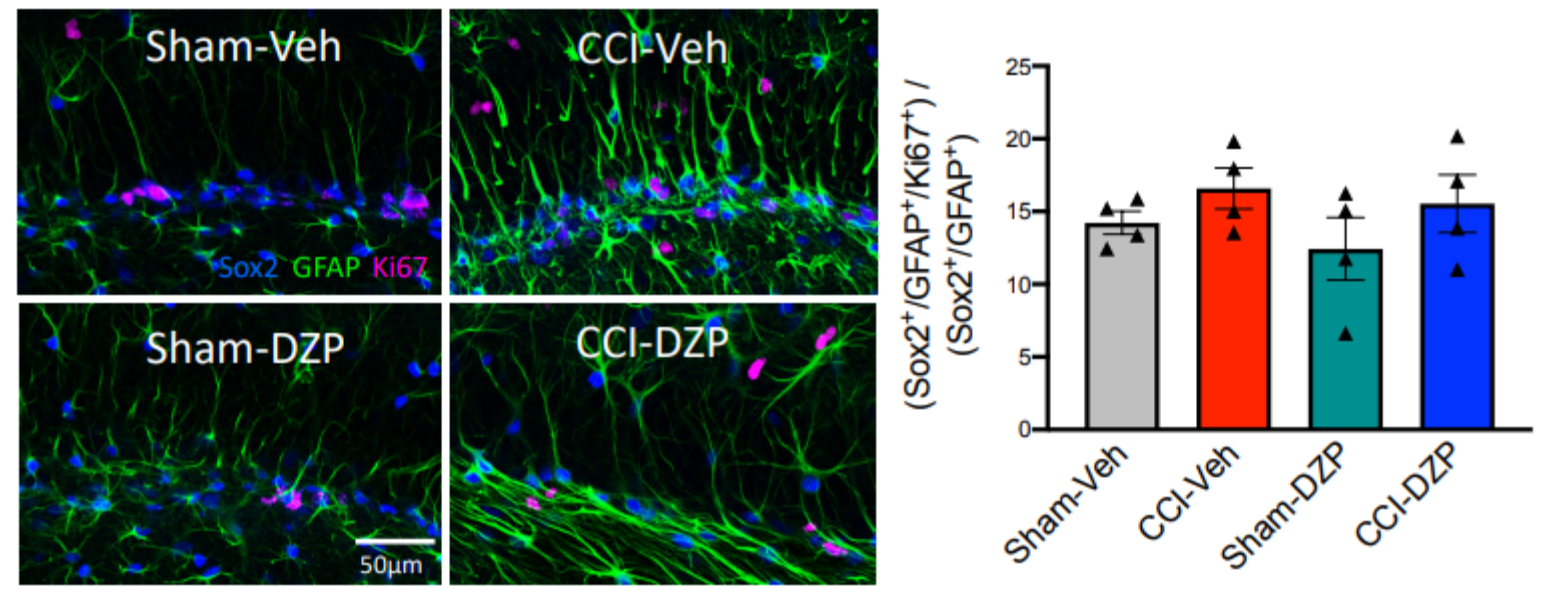

Figure 3: (Left) Representative images of a part of the granular layer across the four experimental treatments 3 days after injury. Sox2 labels neural stem cells and astrocytes. GFAP labels neuronal dendrites and NSC processes. Ki67 labels proliferating cells. (Right) Percent of proliferating neural stem cells (Sox2/GFAP/Ki67) within the total population of neural stem cells (Sox2/GFAP). No significance was found among the groups. 